\title{
EPR study of the symmetry breaking effect in ferroelectric cesium dihydrogen phosphate doped with $\mathrm{Cr} 5+$ ions
}

\author{
Authors: S. Waplak \& V. Hugo Schmidt
}

NOTICE: this is the author's version of a work that was accepted for publication in Solid State Communications. Changes resulting from the publishing process, such as peer review, editing, corrections, structural formatting, and other quality control mechanisms may not be reflected in this document. Changes may have been made to this work since it was submitted for publication. A definitive version was subsequently published in Solid State Communications, VOL\# 52, ISSUE\# 8, (DATE)] DOI\# 10.1016/0038-1098(84)90394-6.

S. Waplak and V.H. Schmidt, "EPR study of the symmetry breaking effect in ferroelectric cesium dihydrogen phosphate doped with $\mathrm{Cr} 5+$ ions," Solid State Communications. 52, 709-712 (1984).

\section{http://dx.doi.org/10.1016/0038-1098(84)90394-6}

Made available through Montana State University's ScholarWorks scholarworks. montana.edu 
BPR STUDY OF THE SYMUETRY BREAXING EFFECT IN FERRORLECTRIC CESIUM DIHYDROGEN PHOSPHATE DOPED WITH $\mathrm{Cr}^{5+}$ IONS

\author{
S. Wap1 ak* and V. Hugo Schmidt
}

Department of Physics, Montana State University, Bozeman, MT 59717, U.S.A.

(Received July 191984 by H. Suh1)

\begin{abstract}
The $\left(\mathrm{PO}_{4}\right)^{3-}$ anits in a $\mathrm{CsH}_{2} \mathrm{P}_{4}$ (CDP) crystal were replaced in a small fraction of sites by $\left(\mathrm{CrO}_{4}\right)^{3-4}$ groups and the EPR of the $\mathrm{Cr}^{5+}$ conter was investigated. Splitting of the EPR 1 ine appears at $T^{*}=245 \mathrm{~K}, 91 \mathrm{~K}$ higher than the ferroelectric transition temperature $T_{c}=154 \mathrm{~K}$. The electronic wave function of $\mathrm{Cr}^{5+}\left(3 \mathrm{~d}^{1}\right)$ is identified os $d_{-}{ }^{2}$. The d $x^{2}-y^{2}$ function couples with the near protons and the reorientation of this unit in the two possible configurations occurs in the paraelectric phase and breaks the symmetry far above $T_{c}$. The observed correlation time $10^{-9}$ sec and a sociated activation energy $\Delta \mathrm{J}=0.215$ oV are discussod.
\end{abstract}

\section{Introduction}

Cesium dihydrogen phosphate, $\mathrm{CsH}_{2} \mathrm{PO}_{4}$ (CDP), is we 11 known as typical example of a hydrogen-bonded ferroelectric compound. Diffuse neutron scattering reveals the existence of onedimensional ordering of the hydrogen bonds parallel to the ferroelectric b aris. 1 The crystal structure of CDP at room temperature has been determined by Uesu and Kobayashi, 2 and by Matsunaga, Itoh and Nakamura. They showed that the symmetry is monoclinic ith space group $P 2 / m$ and two formula units per unit cell. In the ferroelectric phase the space group changes to $P 2_{1}$.

There are two kinds of one-dimensional chains of $\left(\mathrm{PO}_{4}\right)^{3+}$ ions connected by hydrogen bonding. ${ }^{2}$ One chain runs along the $\underline{b}$ axis and the other along the $c$ aris. The phosphate tetrahedron $\mathrm{PO}_{4}$ is deformed from a regular tetrahedron with the following distances between phosphorus and oxygen a toms:

$$
\begin{aligned}
& \text { P- } 0_{1}=1.607 \mathrm{~A}^{\circ}, \quad \mathrm{P}-0_{2}=1.464 \mathrm{~A}^{\mathrm{o}} \text {, } \\
& P-0_{3}=1.539 A^{\circ} \text { and } P-0=1.539 A^{\circ} \text {. }
\end{aligned}
$$

The length of the $0-H(1)$...0 bonds along the caxis is $2.562 \mathrm{~A}^{\circ}$ and they are already ordered at room temperature whereas the $0-H(2) \ldots 0$ bond 1 ength along the ferroelectric baxis is $2.427 \mathrm{~A}^{\circ}$ and these protons are disordered.

This result reflects the fact that a dielectric anoma1y in CDP is only observed along the b axis. 4 Iwata, Koyano and Shibuya 5 report a structure determination of the 1 ow temperature phase of CDP by neutron diffraction with discussion of the transition mechanism derived from the resultant atomic shifts. They found besides

* On leave from Institute of Molecnlar Physics. Po1 ish Academy of Sciences, Poznan, Poland. the hydrogen ordering soveral deviations of other toms from their symmetric positions in the paraelectric phase. The displacement of the $O(1)$ oxygen is found to be consequence of a small rotation of the $\mathrm{PO}_{4}$ totrahedron around the $\mathrm{P}-\mathrm{O}_{2}$ bond. The angle of rotation was estimated to be about $3^{\circ}$. Next is a displacement of the $P$ a tom $10 \mathrm{ng}$ the $\underline{b}$ direction by $0.06 \mathrm{~A}^{\circ}$. These displacements might be responsible for generation of polarization, but the value of polarization ovaluated from this model did not givo good agreement with the experimental value.

In this paper we present our EPR investigation of Cr as a dopant in a CDP crystal. There are two reasons why the $\mathrm{Cr}^{5+}$ probe was chosen. First, the "formal" lonic charge of the $\mathrm{Cr}^{5+}$ ion is the same as that of the replaced unit $P$, and it is near the anion site were moving of protons 18 expected. Second, the $\mathrm{Cr}^{3+}$ probe introduced by Dalal and coworkers 6,7 and Müller and Berlinger into KDP family crystals has provided controversy regarding what kind of phenomena $\mathrm{Cr}^{5+}$ EPR spectra reflect, 1.0., local dynamics of ferroelectric clusters in the pargelectric phase or Halperin-Varma-type centers.

Becanse of the simpler proton ordering in a two-dimensional hydrogen-bond network expected to clarify this problom in CDP:Cr $5+$ crystals.

\section{Experimental Procedure}

Single crystal of CDP were grown by ovaporation from saturated water solution prepared by mixing $\mathrm{Cs}_{2} \mathrm{CO}_{3}$ and $\mathrm{H}_{3} \mathrm{PO}_{4}$ with the nolar ratio 1:2 together with 0.06 mol $\$$ of $\mathrm{x}_{2} \mathrm{CrO}_{4}$.

Tho crystis were lrradiated ${ }^{2}$ th $40 \mathrm{kV} / 10 \mathrm{~mA}$ molybdenum source for 4 hours at roor temperature. The EPR spectra were obtained using a Varian $X$-band spectronetor with $100 \mathrm{kHz}$ modulation and a gas nitrogen system for temperature control and stabilization.

The orthogonal axes for the EPR measure- 
ments was chosen as $\underline{a}, \underline{b}$ and $\underline{c}$." With $\underline{c}$ " porpendicular to the ab plane of the crystallographic axis system chosen by Uesu and Kobayashi. ${ }^{2}$

\section{Experimental Results}

At room temperature, after measuring anisotropy in the throe perpendicular planes, we found that the principal axis g/l lies in the ab plane and makes an angle of $45^{\circ}$ with the b axis. Only one type of $\mathrm{Cr}^{5+}$ is observed at room tempersture.

The spin-Hamiltonian used in describing the EPR spectron has the form

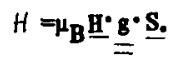

Becanse the spectrum has axial symmetry for rotation around the $g$ || principal axis of the crystal field, the g parameter is described by the expression

$$
g^{2}(\theta)=\left.g\right|^{2} \cos ^{2}(\theta)+\left.g\right|^{2} \sin ^{2}(\theta) .
$$

Here $\theta$ is the angle between 8 |l and the external megnetic field. No change in EPR spectrum was observed in the 100 to $300 \mathrm{~K}$ temperature rogion wen the crystal was rotated round

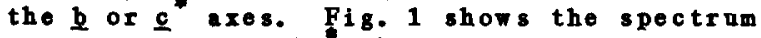
anisotropy in the bc plano at room temperature and $160 \mathrm{~K}$. This $f$ ignre is plotted in the $r$, coordinate system where 6 is the angle between

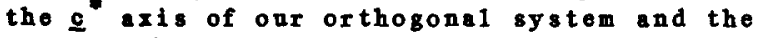
magnotic field which 1 ios along the r-axis.

As is shown in Fig. 1 the single EPR 1 ine (at $300 \mathrm{~K}$ ) is split (at $160 \mathrm{~K}$ ) into two 1 ines wose $g_{z z}$ components 1 ie $\pm 45^{\circ}$ from tho b axis in the be plane. Rotation of the crystal around the [011] direction gives is the $g_{x x}$ and gy compononts of the $g$ tensor at 1 ow temperathre (Tab1e I).

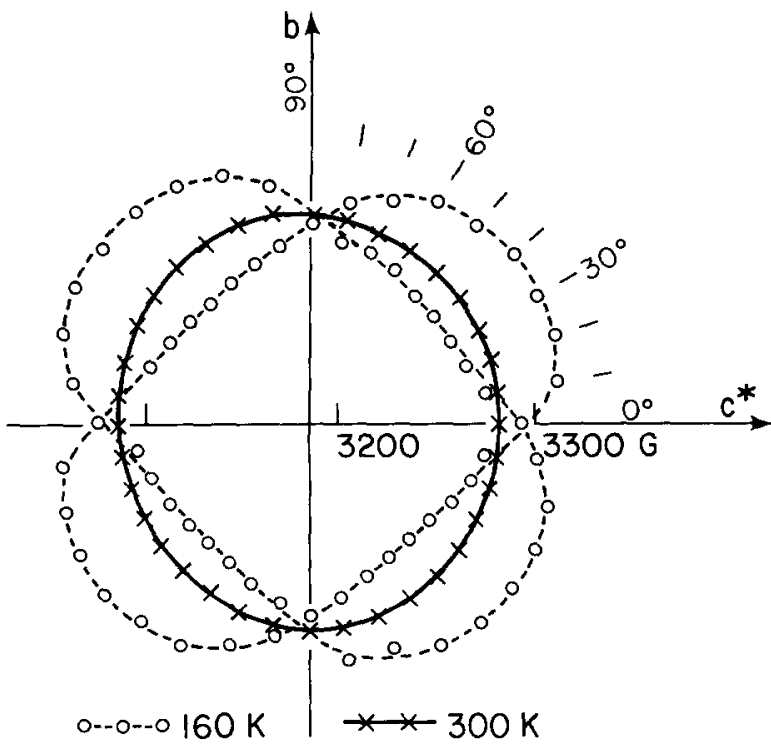

Figure 1

EPR spectrum anisotropy at room temperature (fall line) and $160 \mathrm{~K}$ (broken 1 ine). Because of symetry the EPR splitting can be observed only in the a rotation.
TABLE I

g at $300 \mathrm{~K}$ direction cosines

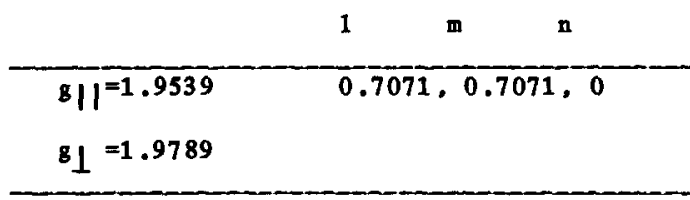

g at $160 \mathrm{k}$ direction cosines

1 m $\quad$ n

$\mathrm{g}_{\mathrm{zz}}=1.9452 \quad 0, \pm 0.7071, \pm 0.7071$

$g_{x x}=1.9764 \quad 0, \mp 0.7071, \pm 0.7071$

$8_{\mathrm{yy}}=1.9848 \quad 1, \quad 0, \quad 0$

The EPR 1 ine splitting versus temperature was measured with the magnetic field parallel to the [011] direction (Fig. 2). Splitting of the EPR 1 ine appears at $T_{c}^{*}=245 \mathrm{~K}, 91 \mathrm{~K}$ higher than the ferroelectric transition temperature.

We have used the line splitting temperature dependence to evaluate the correlation time of proton movement along the hydrogen bonds. From the Bloch equation modified by random jumps between two equaliy probable sites one finds 10 the complex frequencies $\omega_{ \pm}$of the doublet components as

$$
\omega_{ \pm}=i(\Gamma+\gamma) \pm\left(\delta^{2}-\Gamma^{2}\right)^{1 / 2},
$$

where 28 is the maximum doublet splitting. $\Gamma$ is the jump frequency between the two sites, and $\gamma$ is the width of the individual components in the absence of $j$ umps.

The real part of Eq. (3) gives the position of the two doublet components relative to the center of the doublet and the imaginary part

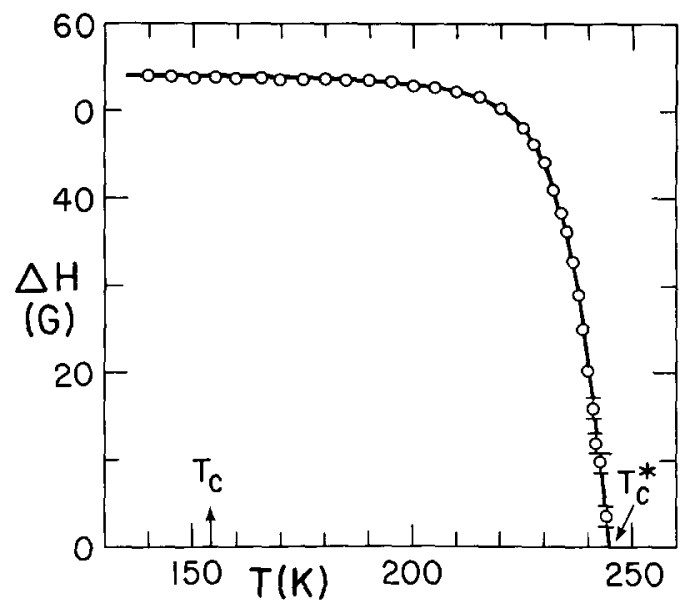

Figure 2

The EPR line splitting $\Delta H$ ys. temperature $T$ for external field $H_{0}$ directed along [110]. 
gives their ridth. When $\Gamma<\delta$ the singlet 1 ine change into a doub1et.

The jump frequency obtained from Eq. (3) is

$$
\Gamma=0.5\left[(28)^{2}-(\Delta \omega)^{2}\right]^{1 / 2} \text {, }
$$

or

$$
\Gamma=\left(g \mu_{B} / 2 t\right)\left[\left(\Delta H_{0}\right)^{2}-\left(\Delta H_{t}\right)^{2}\right]^{1 / 2} .
$$

where $\Delta \mathrm{H}_{\mathrm{O}}$ is the maximum doublet splitting value in gass and $\Delta H_{t}$ the splitting at given temperature.

Taking into consideration the $g=1.967$ value of the center of the doublet we have the following expression for the jump frequency in our experiment:

$$
\left.\Gamma=8.65 \times 10^{6}\left[\Delta \mathrm{H}_{0}\right)^{2}-\left(\Delta \mathrm{H}_{\mathrm{t}}\right)^{2}\right]^{1 / 2} \text {. }
$$

The plot of $\ln \Gamma \mathrm{vs}, 10^{3} / \mathrm{T}$ shown in Fig. $3 \mathrm{can}$ be described by the simple Arrhenius 1 ar

$$
1 / \tau_{c}=\left(1 / \tau_{c o}\right) \exp (U / k T) \text {. }
$$

where $1 / \tau_{c}=\Gamma, 1 / \tau_{c o}=1.38 \times 10^{13}$ sec and $\Delta U=0.215$ eV exceptin the narrow region about $5 \mathrm{Kbelow}$ $T^{*}$. The critical slowing down effect in CDP was measured by kanda et al ${ }^{11}$ in the frequency region below $1 \mathrm{GHz}$. The independent-dipole relaxation time, which is governed by the hopping of protons between the donble well potential minima in the high temperature region, was $\tau_{0}=1.910^{-13}$ sec. They get the $\tau$ value of the ferroelectric relaxation mode by the following expression which is validfor pseado-one dimensional Ising system above $T_{c}$ :

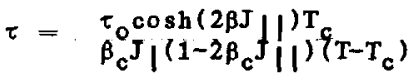

For CDP the observed temperature dependence of the relaxation time wa fitted with she fol1 owing parameters: J J//k=234 $\mathrm{K}$ is the intrachain interaction constant. $J / / k=6.78 \mathrm{~K}$ is the interchain interaction constant, $\beta=1 / \mathrm{kT}$, and $\beta_{c}=1 / \mathrm{kT}$. By using Eq. (9) we obtain in the 200 to $245 \mathrm{~K}$ temperature region the range of values

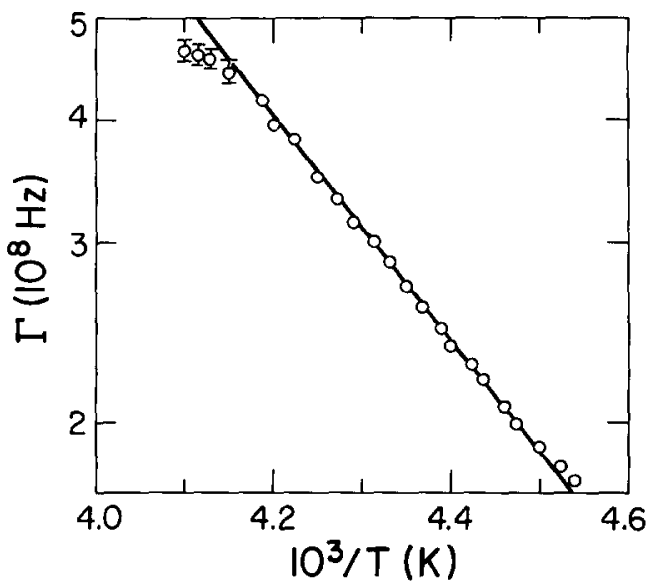

Figure 3

The $1 \mathrm{n} \Gamma \underline{\mathrm{ys}} \cdot 10^{3} / \mathrm{T}$ plot. $\tau_{c}^{-1}=(0.58-1.70) \times 10^{11} \mathrm{sec}^{-1}$ to compare with our experimental value range $\tau_{c}^{-1}=(3.3-4.6) \times 10^{8}$ sec $^{-1}$.

The change of the $g_{z z}$ directions by $\pm 45^{\circ}$ from the b axis requires a corresponding reorientation of the $\mathrm{CrO}_{4}$ tetrahodron gr reorientation of the $d_{x^{2}}{ }^{2}$ orbital of the $\mathrm{Cr}^{3+}$

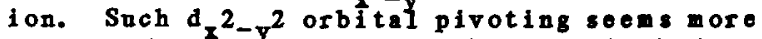
reasonable and has been fell established by ENDOR and $\mathrm{EPR}$ data for $\mathrm{Cr}^{5+}$ in $\mathrm{KH}_{2} \mathrm{AsO}_{4} 0^{8.12}$

Fig. 4 shows the hypothetical pivoting of the ${ }^{2}{ }^{2}-q^{2}$ orbital due to interaction of tho negatively charged orbital with the positive protons.

The relation $g / \mid<g$ between tho exporimental values of the g-tensor components leads to the conclusion that the $d^{2}-y^{2}$ orbital of the $\mathrm{Cr}^{5+}$ ion has 1 owest energy ${ }^{8} \mathrm{and}^{2}$ is perpendicular to the $g$ ll direction above $T_{c}^{*}$. Above $T_{c}^{*}$ the 8 axis lies along the [110] direction bat below $t_{c}$ it 1 ies long the [011] and [011] directions. Such changing of the principal axes requires piroting of the $y^{2}$ lobe around the $c^{\text {axis due }}$ to the interaction with the protons inc-aris bonds which are ordered above $T$.

Dee to coupling of the d $x^{2}-y^{2}$ orbital to the nearest two protons we observe in tho EPR spoctrum a symetry breaking offect whon the frequency of reorientation of the $\mathrm{Cr}^{5+}$ centers associated with b-bond proton jumping is 1 ower than our EPR f requency.

Discussion

Mach interest has recently centered on the possible existence of dynamic contral poak (CP) near structural and ferroelectric phaso transitions. There are two points of view on the physical origin of CP phenomona. First, some have ascribed the CP dynamics to pare 1attice excitation, 13,14 that is, an intrinsic phenomenon. In accord with this view, when the transition is approached from above $T_{c}$, rogions

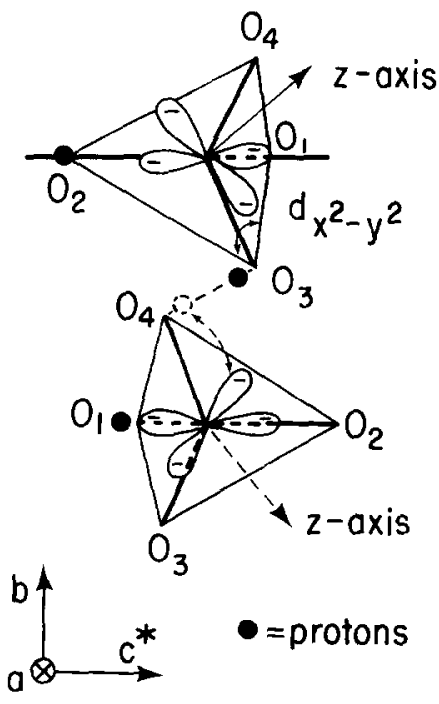

Figure 4

Schematic reorientations of $d_{x^{2}}-y^{2}$ orbital due to moving in hydrogen bonds. 
- ith the short range order and symmetry of the 10w temporature phase start to appear. The other possibility is that the CP mode 1 s due to prosence of impuritios or other dofocts. 15

Halporin and Varnag considered model in which the distortion in defect onit cell may fump betwoen orientations which break symmetry, - situation they describe as a relaxing defect co11. The first EPR identification of this kind of conter was made by Müller ot $1 .^{8}$ for $\mathrm{Cr}^{5+}$ in a $\mathrm{KH}_{2} \mathrm{AsO} 4$ crystal.

Within the relaring halperin-Varma model the reorientational correlation time $\tau$ of the dofect is related to the central peak width $\Gamma$ by the relation

$$
\Gamma=\omega_{8}^{2} / \tau\left(\omega_{8}^{2}+b^{2}\right)
$$

where $\omega^{2}=A\left(T-T_{c}\right)$ is the soft mode frequency and $b^{2}$ is proportional to the defoct concentration. $A$ dof ect concentration of order $10^{-5}$ is sufficient for explaining the CP width in this mode 1.9 In our experiment the fractional $\mathrm{Cr}^{5+}$ concentration was about $10^{-3}$. However, $\Gamma$ cannot be fitted by Eq. (10) but rathor by a simple activation process for which $\Gamma=1 / \tau$.

On the other hand the presence of ferroeloctric olustors far above $T$ with size of order $10^{2} \mathrm{~A}^{\circ}$ in CDP has been woil established by a netron scattering experiment.

It is our opinion that the ferroelectric clusters far above $T_{c}$ are "pinned"' and stabilized by the defect center and by the height of the barrier for the b-bond protons which are coupled with the $d^{2}-r^{2}$ orbital.

As result the jump frequency for the protons interacting with the defect is about $10^{3}$ times 1 ower than in undisturbed parts of the crysta1. The defoct center and associated pinned cluster are switched by the arrival of clusters of an opposite polarity and not by the soft mode fluctuation.

A similar explanation has been made by

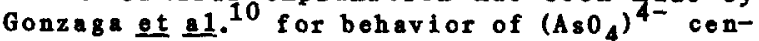
ters in ADA-ADP mired crystals.

The values obtained for $\tau$ and $\Delta U$ for CDP:C $r^{5+}$ as we11 as the f1attening of the $1 \mathrm{n} 1 / \tau$ versus $10^{3} / T$ plot near $T_{\text {are quite similgr to }}$ the results obtained by lamotte et $1 .^{16}$ for $\left(\mathrm{AsO}_{4}\right)^{4-} \mathrm{radicals}$ in KDA. The flat portion of the in $\Gamma$ curve in our case with the activation energy $\Delta U=0,05 \mathrm{eV}$ and typical $\tau_{0}=10^{-12} \mathrm{sec}$ value gives $\tau=10^{-11}$ sec and can be connected with of $f-$ center motion of $\mathrm{Cr}^{5+}$ ions along the bax which can explain the nonaxial g tensorbelow $\mathbf{T}_{\mathrm{c}}^{*}$

It follow that the cooperative dynamics around $\mathrm{Cr}^{5+}$ cannot be intrinsic.

Acknow ledgements-- Te thank Professor $J$. E. Drambeller for the use of his EPR apparatas. This work was supported in part by National Science Foundation Grant DMR-8205280.
1. B.C. Frazer, D. Semmingsen, W.D. E11enson and G. Shirane, Phys. Rev. B 20, 2745 (1979).

2. Y. Desu and T. Kobayashi, Phys. Stat. Sol. (a) 3. 475 (1976).

3. H. Matsunaga, $\mathrm{K}$. Itoh and E. Nakamura, J. Phy 8. Soc. Japan 48, 2011 (1980).

4. A. Levstik, R. Blinc, P. Kadaba, S. Cizikov, I. Levstik, and C. Filipic, Solid State Commun. 16, 1339 (1975).

5. I. Iwata, N. Koyano, and I. Shibuya, J. Phys, Soc. Japan 49, 304 (1980).

6. N.S. Dalal and A.H. Redoch, Abstr., 6th Int. Symp. Magn. Reson., 241 (1977).

7. K.A. Müller, N.S. Dalal and W. Berlinger, Phy 8. Rev. Lett. 36, 1504 (1976).

8. K.A. Müller and W. Berlinger, Phys, Rev. Lett. 37. 916 (1976).
9. B. I. Halperin and C.M. Varma, Phys, Rev, B 14, 4030 (1976).

10. L.V. Gonzaga, A.S. Chayes, R. B1inc, G.M. Ribeiro, R. Gazinelli, and G. Rius, Solid Stato Commun. 27, 495 (1978).

11. E. Kanda, A. Tamaki, and T. Fujimara, J. Phys, C 15, 3401 (1982).

12. J. Gail1ard, P. Gloux, and K. A. Müller, Phys. Rev. Lett. 38, 1216 (1977).

13. J. Feder, in Local Properties at Phase Transition (K. A. Müller and A. Rigamonti, Eds.) p. 312 (North-Holland, Amsterdam, 1976).

14. T, Schneider and E, Stol1, Phys. Rev, Lett. 31, 1254 (1973) and 35, 296 (1975).

15. S. M. Shapiro, J. D. Axe, G. Shirane, and T. Riste, Phys. Rev. B 6,4332 (1972).

16. B. Lamotte and J. Gaillard, J. Chem. Phys. 57, 3319 (1972). 